\title{
The role of point-of-care ultrasound in pediatric acute respiratory distress syndrome: emerging evidence for its use
}

\author{
Samantha K. Potter ${ }^{1}$, Michael J. Griksaitis ${ }^{1,2}$ \\ ${ }^{1}$ Pediatric Intensive Care Unit, Southampton Children's Hospital, Southampton, UK; ${ }^{2}$ Faculty of Medicine, University of Southampton, \\ Southampton, UK \\ Contributions: (I) Conception and design: All authors; (II) Administrative support: All authors; (III) Provision of study materials or patients: All \\ authors; (IV) Collection and assembly of data: All authors; (V) Data analysis and interpretation: All authors; (VI) Manuscript writing: All authors; (VII) \\ Final approval of manuscript: All authors. \\ Correspondence to: Dr. Michael J. Griksaitis, MBBS(Hons), MSc, MRCPCH, FFICM. Consultant Pediatric Intensivist, Southampton Children's \\ Hospital, University Hospital of Southampton, Tremona Road, Southampton, UK SO16 6YD. Email: Michael.Griksaitis@uhs.nhs.uk.
}

\begin{abstract}
Pediatric acute respiratory distress syndrome (PARDS) remains an important cause of significant morbidity and mortality. The 2015 PALICC definition of PARDS requires chest imaging to diagnose the presence of new pulmonary infiltrate(s). Traditionally chest radiography or computerised tomography have been used. However, these carry the limitations of exposure to ionizing radiation, need to transfer the critically unwell child, lag-time with clinical correlation and lack of immediate results. The use of point of care ultrasound (POCUS) has been well established in adult emergency medicine and critical care. Furthermore, the adult literature clearly demonstrates that lung POCUS is a safe and validated tool, which is highly sensitive and specific when compared to chest radiography for differentiating the causes of respiratory failure, including ARDS. Whilst pediatric specific data is limited, it has been shown that the signs seen in adults are reproducible in critically ill neonates and children. Furthermore, the numerous benefits of POCUS in the paediatric setting are compelling and include lack of ionizing radiation, immediate feedback, promoting time at the bedside of the critically unwell child, and ease of serial assessments. This review article presents the emerging evidence demonstrating that lung POCUS can be used not only to support the diagnosis of pediatric ARDS, but also to assess for complications, monitor progression and thus guide management. We hope it will stimulate much needed collaborative research into this exciting field of imaging and its applications to PARDS and beyond.
\end{abstract}

Keywords: Point of care testing; ultrasound imaging; respiratory distress syndrome

Submitted Feb 24, 2019. Accepted for publication Jul 15, 2019.

doi: $10.21037 /$ atm.2019.07.76

View this article at: http://dx.doi.org/10.21037/atm.2019.07.76

\section{Introduction}

Point of care ultrasound (POCUS) is widely used and supported by the literature throughout adult emergency medicine and critical care. It is an efficient, non-invasive imaging modality that allows rapid, serial assessments of patients to guide diagnosis and review immediate interventions at the bedside by the attending clinician. This review will present the emerging evidence to provide a compelling argument for the use of POCUS in pediatric ARDS from diagnosis to follow up.

\section{POCUS in pediatrics}

The use of POCUS in pediatrics is gaining momentum, and whilst pediatric specific data remains limited when compared to adult critical care, there is growing pediatric evidence demonstrating the high sensitivity and specificity of lung POCUS in the diagnosis of pneumonia, bronchiolitis, pleural effusion and pneumothorax (1). POCUS confers many advantages in the pediatric arena; no radiation allows safe and recurrent assessment of the child's pathology and impact of treatment. Furthermore, it 
promotes time at the bedside of the critically ill child, which has direct benefits of noting clinical changes promptly (2), and potentially indirect benefits of increasing parental satisfaction. In their study on bedside ultrasound in the adult emergency department and patient satisfaction, Howard et al demonstrated a statistically significant higher overall patient satisfaction in the group who received bedside ultrasound (mean difference 0.46 with a confidence interval of $95 \%$ CI, 0.17-0.75) (3). Given that POCUS promotes time at the bedside with the child and family and offers immediate feedback, it is highly likely to stimulate communication and thus foster improved relationships between the clinical team and family.

\section{POCUS in respiratory assessment}

Lung POCUS has been shown to have significant impact on clinical decision-making, and be superior to chest radiography when assessing the critically unwell dyspnoeic adult patient $(4,5)$. It has been documented extensively throughout the literature that lung ultrasound confers higher sensitivity and specificity when diagnosing consolidation, interstitial syndrome and pleural effusions in all age groups $(1,2,4,6,7)$. Furthermore, compared to chest radiography, lung POCUS can be performed quickly with minimal movement of the patient and provide immediate results to inform management. The ease of repeated scans then allows the attending clinician to assess the response to interventions and inform on going management (2).

In their retrospective study of 244 critically unwell adult patients with dyspnoea, Saigal et al. developed a classification system based on combined lung and cardiac ultrasound findings and arterial blood gas analysis. They demonstrated that dyspnoeic patients could be classified into seven groups; firstly, lung ultrasound was used to determine the presence of B lines (wet lungs, or alveolar defect) or A-lines (dry lungs). These appearances were then coupled with blood gas analysis to verify whether the presence of hypoxia; hypoxia and hypercarbia; or no hypoxia (Figure 1) (4). The combined cardiac and lung ultrasound technique, created by Lichtenstein and Mezière in the Blue Protocol (8) was then used to classify whether the presence of wet lungs and hypoxia were secondary to consolidation, i.e., pneumonia, or alveolar oedema. They found that the sickest adult patients were those with a lung ultrasound pattern of either alveolar defect-consolidation (B lines and hypoxia), or perfusion defect (A lines and hypoxia). Furthermore, by classifying the cause of $\mathrm{B}$ lines as either consolidation or oedema, they were able to identify that the former group benefited from intubation and the latter from non-invasive ventilation (NIV). Therefore, the authors were able to conclude that this combined approach allowed critically unwell dyspnoeic patients to be classified in an organised manner, thus identifying and prioritizing the sickest patients in need of immediate intervention (4).

Furthermore, a prospective study by Xirouchaki et al. in 2014 (5) analysed 189 adult patients in whom lung ultrasound was requested either due to unexpected deterioration in arterial blood gas, or because of suspected pathology such as pneumothorax, pleural effusion, unilateral atelectasis, pneumonia, or diffuse interstitial syndrome. They found that of the 253 lung ultrasound performed, 119 (47\%) provided information that led to a direct change in management. The majority [81] of these interventions were invasive, such as thoracocentesis, bronchoscopy or hemofiltration. The remaining 38 interventions were noninvasive and included a change in PEEP, recruitment manoeuvres, initiation/change of antibiotics and use of diuretics. They also found that 53 of the lung ultrasound examinations revealed diagnoses not previously suspected by the diagnosing clinician, for example pleural effusion, pneumothorax and pneumonia. The combination of these findings led the authors to conclude that, "lung ultrasound has a significant impact on clinical decision making and management" (5).

From this literature we have demonstrated that lung POCUS alone, or in combination with cardiac POCUS, is very helpful to differentiate causes of respiratory failure and determine whether alveolar oedema is secondary to acute cardiac failure or primary lung pathology. Although many of these studies are carried out in adult patients, the ultrasound findings should be similar in pediatric patients. We will now look more specifically at acute respiratory distress syndrome (ARDS) and the role of POCUS.

\section{Pediatrics acute respiratory distress syndrome (PARDS) \& respiratory imaging}

In 2015, the Pediatric Acute Lung Injury Consensus Conference (PALICC) set out to identify differences between adults ARDS and PARDS, provide recommendations and stimulate interest in future research priorities.

The definition of PARDS, similar to adult ARDS, is based on several factors including: timing of onset of hypoxemia, respiratory failure not fully explained by fluid overload, the presence of new infiltrates on chest imaging 




Figure 1 Classification on the basis of lung ultrasound and blood gas analysis in dyspnoeic critically ill patients (4).

consistent with pulmonary parenchymal disease and markers of oxygenation depending on whether the child is receiving invasive [oxygenation index (OI)] or NIV $\left[\mathrm{PaO}_{2} /\right.$ $\mathrm{FiO}_{2}$ (PF ratio) or the $\mathrm{SpO}_{2} / \mathrm{FiO}_{2}$ (SF ratio)] (9). The main differences to the adult definition include using $\mathrm{OI}$ and oxygenation saturation index (OSI) instead of PF ratio, and removing the need for "bilateral infiltrates" to be seen on chest imaging, and replacing this with the presence of "new infiltrate(s) consistent with acute pulmonary parenchymal disease" (9). This last change was made based on the lack of evidence that patients with unilateral compared to bilateral pulmonary infiltrates have different aetiology, treatment or outcomes (9). Figure 2 summarises the differences in imaging requirements for PARDS and ARDS.

Chest imaging is therefore a requirement for the diagnosis of PARDS, however there is no mention of the preferred modality of imaging in the PALICC recommendations. Point of care lung ultrasound has multiple benefits including immediate results, promoting time at the bedside of a critically ill child, ease of repeat scans to assess and guide immediate management, high sensitivity and specificity when compared to chest radiography, affordability and lack of ionizing radiation $(1,2,10)$.

Lung POCUS and ARDS is well described in adults $(6,8,10)$. The current PARDS definition requires 'chest imaging' to diagnose PARDS by the attending clinician. We will now discuss how lung POCUS can help you diagnose PARDS and potentially fulfil the chest imaging requirements of the definition.

\section{Normal lung POCUS features}

Before considering the lung POCUS features of PARDS, we will look at the normal lung POCUS. Full descriptions of how to scan the respiratory tract and use lung POCUS in pediatrics has been published elsewhere (2). Figure 3 


\begin{tabular}{|c|c|}
\hline \multicolumn{2}{|c|}{ Imaging criteria for diagnosing ARDS } \\
\hline Pediatric ARDS & Adult ARDS \\
\hline $\begin{array}{c}\text { Chest imaging (modality not } \\
\text { specified) }\end{array}$ & $\begin{array}{c}\text { Chest radiograph or computerized } \\
\text { tomography }\end{array}$ \\
\hline New infiltrates, occurring acutely & Bilateral opacities \\
\hline $\begin{array}{c}\text { Consistent with parenchymal } \\
\text { disease }\end{array}$ & $\begin{array}{c}\text { Not fully accounted for by pleural } \\
\text { effusions, collapse or nodules }\end{array}$ \\
\hline
\end{tabular}

Figure 2 Imaging diagnostic criteria for acute respiratory distress syndrome (ARDS) in children and adults.

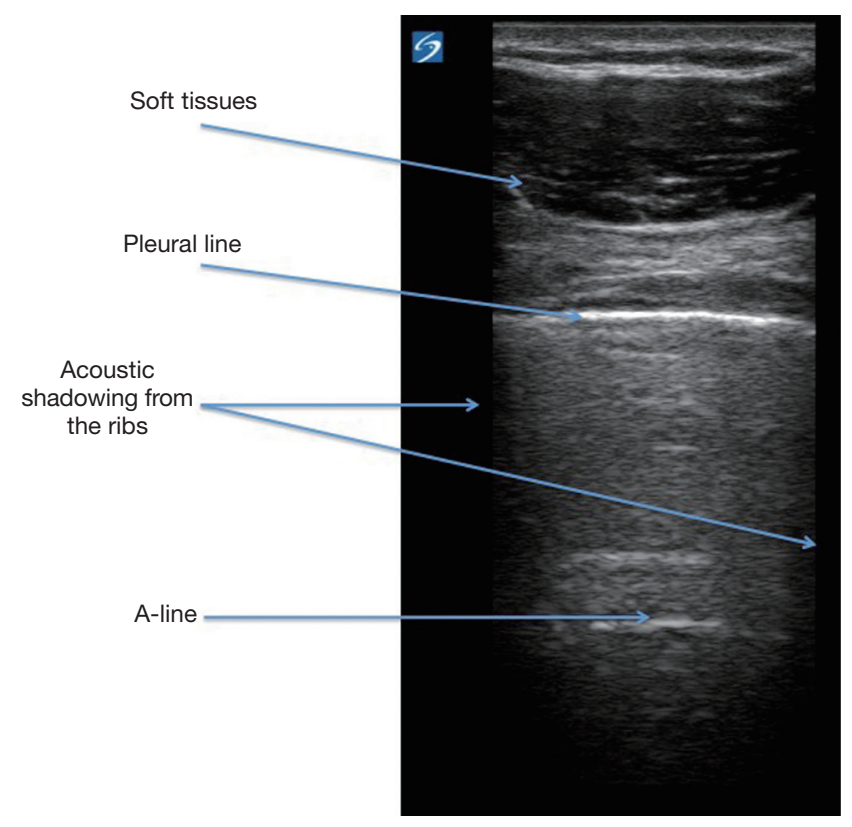

Figure 3 Normal lung point of care ultrasound (POCUS) anatomy on an infant with a linear probe.

summarises the key normal findings on lung POCUS. The authors' experience is that using a high frequency linear probe provides very good image quality and depth. The use of a lower frequency curvi-linear probe maybe needed in larger children when further depth is required, but at the expense of image quality.

Start by identifying the Bat-wing sign, which is described because of the appearances of the upper and lower adjacent ribs producing 'the wings of the bat' with the pleural line forming the back (body) of the bat (2). The pleural line is a dense, crisp sharp white line, and should be seen shimmering and sliding back and forth to indicate normal movement of the pleura. The absence of pleural sliding suggests pathological processes, either because of lung under ventilation (e.g., collapse-consolidation, endobronchial intubation) or because of separation of the visceral and parietal pleura by air (i.e., a pneumothorax) (2). Pleural sliding, or indeed the absence of, can be confirmed by using the 'motion mode' (M-mode). This plots movement against time and allows movement of a given structure to be assessed; in lung POCUS this structure is the pleura. In M-mode, pleural sliding creates a seashore sign; the static muscle/soft tissue allows passage of the ultrasound which then hits the pleura, which if moving causes disruption of the ultrasound and therefore disrupts the M-mode trace (Figure 4) $(2,11)$. Finally, the normal lung ultrasound will demonstrate A-lines, which are horizontal white lines and are artefact reflections of the pleural line, and therefore run in parallel to the pleural line (2) (Figure 3).

\section{PARDS-relevant abnormal lung pocus features}

There are many abnormalities that can be seen on lung POCUS; a full review of these is outside the scope of this review. However, we will consider different ultrasound findings relevant to PARDS. We must emphasise that the POCUS findings of PARDS are non-specific and can be found in many other conditions. It is therefore important that a full clinical correlation is made with the POCUS findings.

\section{Interstitial (alveolar) oedema}

B-lines are dense white, vertical lines arising from the pleural line and obliterate the A-lines; they can represent fluid in the alveoli or interstitial thickening (Figure 5A). The presence of multiple B-lines can be seen in pulmonary oedema, ARDS, fibrosis and transient tachypnoea of the newborn $(2,6,10,11)$.

\section{Consolidation \& lung parenchymal disease}

Pulmonary consolidation also produces characteristic POCUS signs, including dynamic air bronchograms, and the tissue-like sign in which the consolidated lung has the appearance of solid viscera (Figure $5 B, C)(2,6)$. The shredsign may also be seen, and demonstrates the border where the area of consolidated lung meets the aerated lung and appears shredded (2). Finally, the lung pulse represents movement of the pleura in relation to transmissions of 




Figure 4 Normal M-mode trace of sliding pleura producing the 'sea-shore' sign in an infant with a linear probe.

A

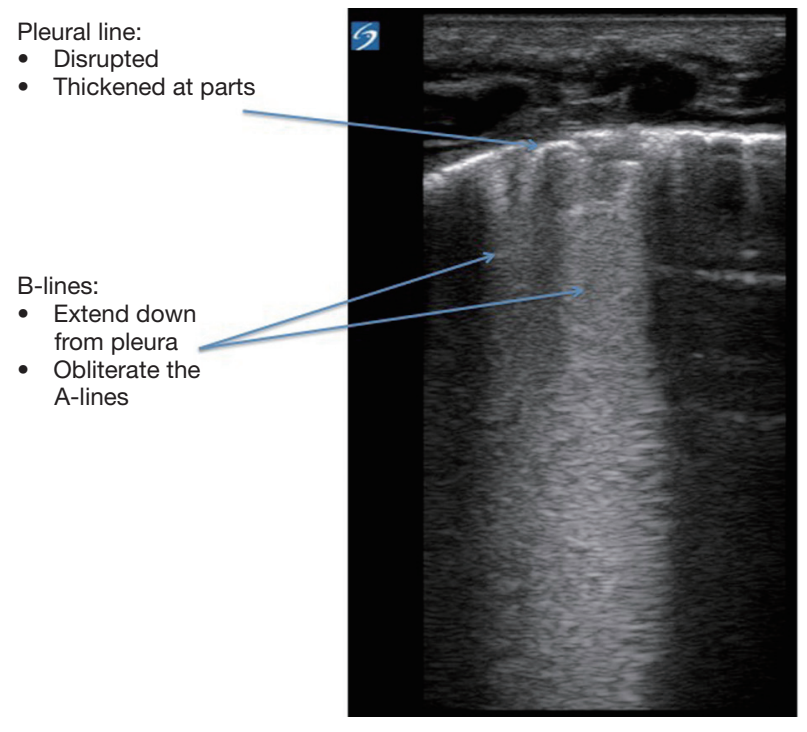

B Pleural line:

- Disrupted

- Thickened at parts

- Not as clear

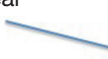

Sub-pleural consolidation

\section{B-lines:}

- Extend down

from pleura

- Obliterate the A-lines

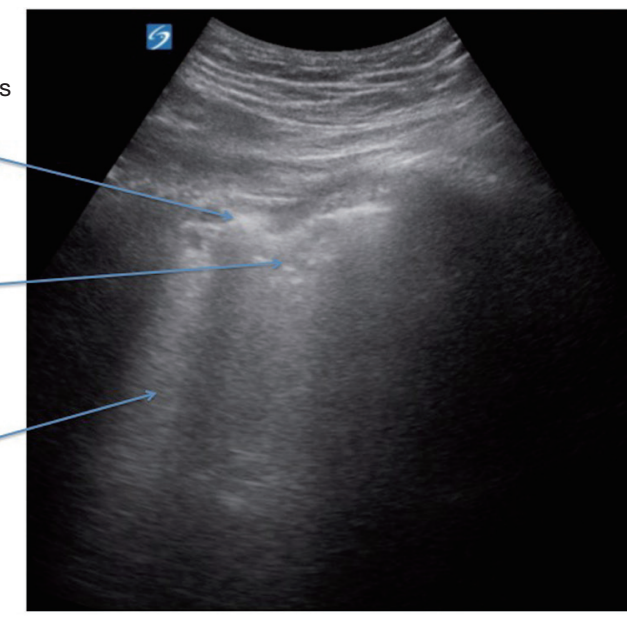

C

Pleural line:

- Thickened

- Not as clear

Air
Bronchograms
\& consolidated

lung tissue

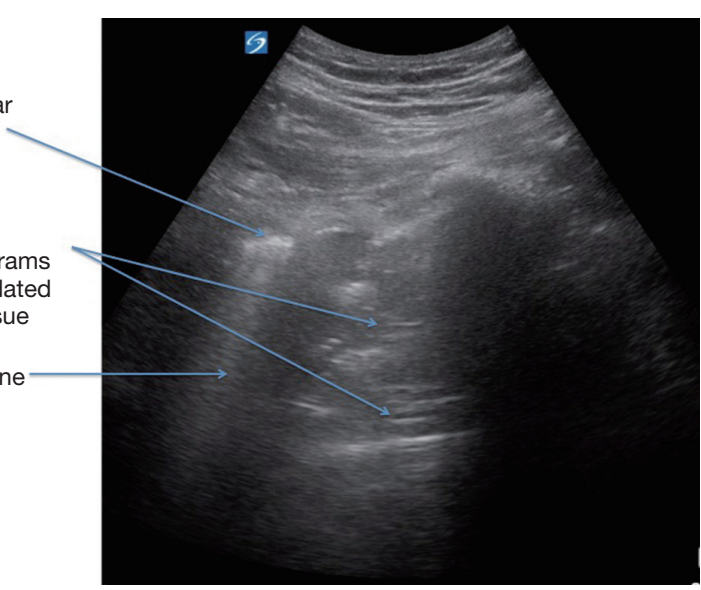

Figure 5 Cases of pediatric acute respiratory distress syndrome (PARDS) as seen with point of care ultrasound (POCUS). (A) Example lung POCUS findings in PARDS (infant with linear probe); (B) example lung POCUS findings in PARDS (teenager with curvi-linear probe); (C) example lung POCUS findings in PARDS (teenager with curvi-linear probe). 

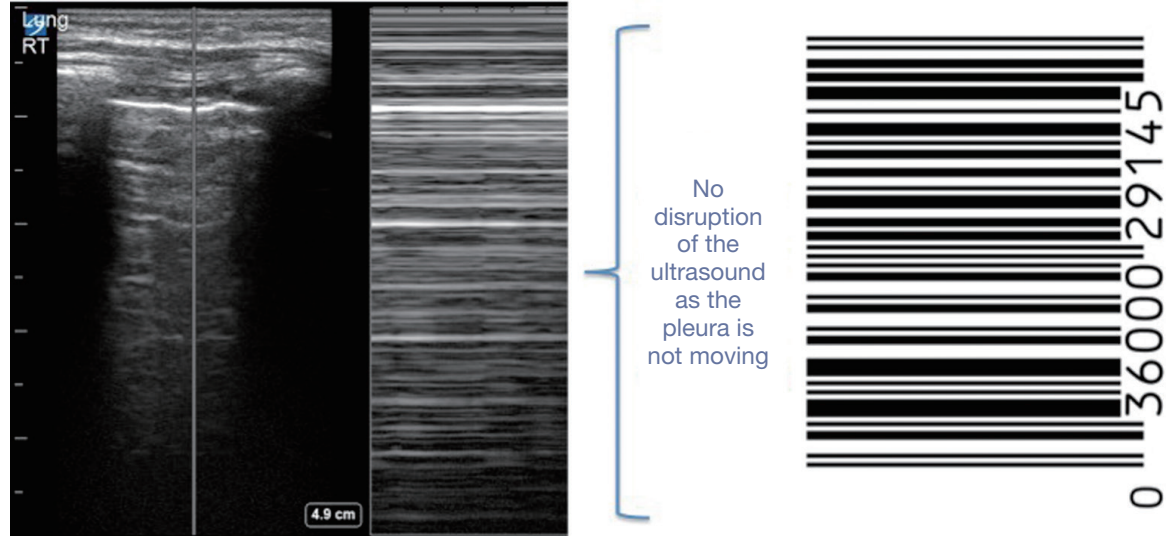

Figure 6 Abnormal M-mode trace of no pleural sliding; producing the 'barcode' (also called 'stratosphere' sign) in an infant with a linear probe.

\begin{tabular}{|c|c|c|c|c|c|c|c|c|c|c|c|}
\hline & $\begin{array}{l}\text { Batwing } \\
\text { sign }\end{array}$ & $\begin{array}{l}\text { Pleural } \\
\text { Line }\end{array}$ & $\begin{array}{l}\text { Pleural } \\
\text { sliding }\end{array}$ & $\begin{array}{l}\text { A- } \\
\text { lines }\end{array}$ & $\begin{array}{l}\text { Sea- } \\
\text { shore } \\
\text { sign }\end{array}$ & B-lines & $\begin{array}{l}\text { Barcode/ } \\
\text { stratosphere sign }\end{array}$ & $\begin{array}{l}\text { Presence } \\
\text { of a lung } \\
\text { point }\end{array}$ & $\begin{array}{l}\text { Tissue-like } \\
\text { sign \& shred } \\
\text { sign }\end{array}$ & $\begin{array}{l}\text { Dynamic Air } \\
\text { bronchograms }\end{array}$ & Lung Pulse \\
\hline Normal lung & $\checkmark$ & $\checkmark$ & $\checkmark$ & $\checkmark$ & $\checkmark$ & $\begin{array}{l}1-2 \mathrm{~B}- \\
\text { lines }\end{array}$ & $x$ & $x$ & $x$ & $x$ & $\begin{array}{l}\text { Can sometimes } \\
\text { be seen }\end{array}$ \\
\hline $\begin{array}{l}\text { Pulmonary } \\
\text { oedema }\end{array}$ & $\checkmark$ & $\alpha$ & 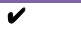 & $x$ & $\checkmark$ & $\checkmark$ & $x$ & $x$ & $x$ & $x$ & $x$ \\
\hline Pneumothorax & $\checkmark$ & 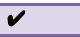 & $x$ & $\checkmark$ & $x$ & $x$ & $\checkmark$ & $\checkmark$ & $x$ & $x$ & $x$ \\
\hline Consolidation & $\checkmark$ & $\checkmark$ & $\checkmark$ & $x$ & $\checkmark$ & $\checkmark$ & $x$ & $x$ & $\checkmark$ & $\checkmark$ & $\checkmark$ \\
\hline $\begin{array}{l}\text { ARDS } \\
\text { (in spared } \\
\text { areas of lung } \\
\text { can be normal) }\end{array}$ & $\checkmark$ & $\checkmark$ & $V / x$ & $x$ & $\checkmark / x$ & $\checkmark$ & $\checkmark / x$ & $x$ & $\begin{array}{l}\text { If coexisting } \\
\text { consolidation }\end{array}$ & $\begin{array}{l}\text { If coexisting } \\
\text { consolidation }\end{array}$ & $\begin{array}{l}\text { If coexisting } \\
\text { consolidation }\end{array}$ \\
\hline
\end{tabular}

Figure 7 Summary of features found on lung point of care ultrasound.

cardiac pulsations. This can be seen most prominently in the consolidated or underventilated lung, and would rule out a pneumothoax $(2,12)$. Particularly in smaller children the lung pulse can be seen in normal lung, especially on the left side.

\section{Absent pleural sliding}

In contrast to the normal 'sea-shore' sign seen in M-mode, absent pleural sliding will produce the appearances of a 'barcode' or 'stratosphere', which represents the lack of pleural movement (Figure 6) (2). The absence of pleural sliding can occur due to under ventilated consolidated lung in PARDS. It can also occur in a pneumothorax and it is obviously important to differentiate the two. There are key features to aid the diagnosis of pneumothorax. These include the absence of B lines. The 'barcode' or 'stratosphere', which confirms the absence of pleural movement, will not contain a lung pulse (i.e., the cardiac impulse will not be transmitted and so not demonstrated on the M-mode trace) (2). It is also important to identify the presence of a 'lung point'. This is the most specific sign, and the only 'rule in' sign to diagnose a pneumothorax. It represents the point at which the visceral and parietal pleural start to separate (2).

Figure 7 summarises all the key features that can be relevant to the diagnosis of PARDS or its complications. Figure 5 demonstrates some key findings seen on lung POCUS in PARDS.

Lung POCUS findings can be considered to:

* Assist the diagnosis of PARDS;

* Assist with identifying complications of PARDS (e.g., air leaks);

* Assist the monitoring of lung improvement/ deterioration.

\section{Lung POCUS in the diagnosis of ARDS}

Lichtenstein first outlined the features of ARDS on lung 
ultrasound as diffuse B line artefacts $(8,10)$. Further features can include the presence or absence of pleural sliding and sub-pleural consolidation (10). The challenge, as with the adult population, is differentiating signs of ARDS from other causes of respiratory failure, in particular pulmonary oedema. Copetti et al. reported features to help distinguish cardiogenic pulmonary oedema from ARDS (13). They found both cohorts of patients displayed alveolar interstitial features (B-lines). However, features that suggested ARDS rather than cardiogenic pulmonary oedema included:

* Abnormalities of the pleural line (e.g., thickening);

* Absence or reduction of pleural sliding;

* Spared areas of lungs (often normal lung POCUS in non-dependent regions);

* Associated consolidation;

* Presence of a lung pulse (as seen on M-mode).

In addition, the acute cardiogenic pulmonary oedema group were more likely to have a pleural effusion than the ARDS group (13).

The location of the POCUS abnormalities can also help to suggest PARDS as a diagnosis. Computerised tomography (CT) in ARDS demonstrates dense consolidation in the most dependent regions (i.e., posterior). Moving more anterior then shows ground-glass appearances, before reaching normal or hyperinflated lung anteriorly. The lung POCUS can reflect this as well, with consolidation seen posteriorly (dependent regions) and then in moving anterior B-lines of differing intensity and subpleural consolidation. There will also be normal lung (known as 'spared areas') in the non-dependent regions.

The adult literature provides excellent examples of incorporating lung ultrasound to aid distinguishing different causes of respiratory failure. In 2008, Lichtenstein and Mezière created the BLUE protocol in which lung ultrasound can be used to rapidly diagnose patients in acute respiratory failure (8).

The aforementioned retrospective study by Saigal et al. (4) developed a classification system based on lung ultrasound appearances and arterial blood gas analysis. This demonstrated, as with other studies, that lung ultrasound has high diagnostic accuracy and is more sensitive and specific when compared to chest radiography at diagnosing consolidation, pleural effusion and interstitial syndrome $(2,4,6)$. Lung ultrasound was used to classify adult patients into those with wet lungs (presence of B lines) and dry lungs (A-lines) and then assessed arterial blood gases to determine presence of hypoxia. Echocardiography was then used to aid differentiation between consolidation and pulmonary oedema. The authors found that this classification helped identify the sickest patients [those with wet lungs (extensive B lines) and hypoxia] and predict need for intervention (4). It was demonstrated that the groups with the highest mortality were patients with perfusion defect (dry lungs and hypoxia) (50\%), although numbers were very small, followed by patients with alveolar-defect consolidation $(21.7 \%)$. This is relevant to the pediatric population in whom the most common cause for PARDS is pneumonia $(9,14)$. Therefore, arguably identifying and prioritising these patients with consolidation, coupled with serial POCUS assessments, will allow for earlier detection of the deteriorating patient and diagnosis of PARDS.

Furthermore, differentiating the cause of B lines into alveolar defect-consolidation or pulmonary oedema had an important role regarding interventions. The highest rate of intubation was $60.9 \%$ in the group of adult patients with alveolar defect-consolidation, i.e., pneumonia, compared to $21.7 \%$ of patients with pulmonary oedema. Similarly, those adults with pulmonary oedema were more likely to require NIV (43.5\%) compared to just $9.2 \%$ of the group with consolidation (4).

Whilst it must be noted that this is a retrospective adult study with small numbers, the results are promising for the use of POCUS in diagnosing the cause of respiratory failure, identifying the sickest patients, and predicting those in need of invasive ventilation. It certainly warrants a larger trial into this area.

Huang et al. also highlight the challenges of diagnosing ARDS in the elderly, co-morbid population with respiratory failure. This prospective study of 51 patients demonstrated a combined cardiopulmonary ultrasound approach provided greater diagnostic accuracy than lung ultrasound alone. Furthermore, it was demonstrated that changes in pulmonary ventilation could be detected on lung ultrasound prior to a fall in $\mathrm{PaO}_{2} / \mathrm{FiO}_{2}$, which could allow for earlier recognition and intervention in ARDS (15). The authors of this review feel that there are similarities with the elderly, fragile patients with multiple comorbidities to some of our fragile pediatric population.

It is clear that lung ultrasound is a safe and validated tool that can be useful in the diagnosis of ARDS, especially when combined with markers of oxygenation and cardiac ultrasound. However, traditionalists may argue why not continue to use chest radiography or chest CT to diagnose the changes needed to diagnose PARDS?

There is increasing evidence for the superiority of ultrasound over chest radiography. In a recent review on 
the use of POCUS to assess respiratory failure in children, Ord and Griksaitis summarised the literature demonstrating increased sensitivity and specificity of lung POCUS in the diagnosis of pneumothorax, pleural effusion, consolidation and pulmonary oedema (2). Marin et al. in their summary of evidence for POCUS, also highlight pediatric specific data suggesting the superiority of lung ultrasound over chest radiography in the diagnosis of pneumonia (1). In their meta-analysis of the use of lung ultrasound for the diagnosis of pneumonia, Pereda et al. concluded that lung ultrasound can be used accurately even by the non-expert (7). Lichtenstein has also demonstrated $93-98 \%$ sensitivity and $78-100 \%$ specificity in the detection of ARDS by lung ultrasound $(6,8)$.

It is only fair to outline the limitations to using POCUS, including limited pediatric data, limited opportunities for training as a pediatrician, dependence on skill of the practitioner, difficulty distinguishing blood from serous fluid, and the impact of body habitus on image accuracy (2). However, in comparison to other imaging modalities, lung ultrasound has no ionizing radiation, provides rapid and serial bedside assessment with real-time feedback, does not require potentially risky transportation, is relatively costeffective, and importantly promotes time at the bedside of the critically unwell child $(2,10)$. We will now consider how lung POCUS can be used beyond the diagnosis of pediatric ARDS.

\section{Lung POCUS in the recognition of complications of ARDS}

Data surrounding the mortality and long-term outcomes of PARDS is scarce. Whilst there is a general trend towards improving mortality, it still remains significant. In a recent systematic review and meta-analysis, overall mortality from PARDS was approximately 24\% (16), however, data provided by Khemani et al. in proceedings from PALICC reported mortality as high as $40 \%$ in pediatric patients with severe hypoxaemia $(\mathrm{OI}>16)(17,18)$. With this is mind it is vital to recognise and treat the complications of PARDS, including but not limited to, pneumothorax, secondary bacterial infection (ventilator associated pneumonia), and ventilator-induced lung injury.

As detailed previously, lung POCUS can be used to accurately diagnose pneumothorax with $87 \%$ sensitivity and $99 \%$ specificity compared to $46 \%$ and $100 \%$ in chest radiography $(2,19)$. The speed, ease, and safe repeatability of lung ultrasound also make it a potentially more rapid, bedside modality to diagnose and then treat this potential complication of PARDS.

Secondary bacterial infection, and ventilator-associated pneumonia (VAP) remain an important cause of mortality and morbidity in any mechanically ventilated patient. Lung POCUS is again superior to chest radiography in terms of sensitivity and specificity for diagnosing consolidation $(1,2,6,20)$. Further pediatric specific data presented by Marin et al. in their summary of evidence for the use of POCUS in pediatric emergency medicine, included an Italian study by Copetti and Cattarossi (21), and a randomised controltrial by Jones et al. (22) that again support the use of lung ultrasound in the diagnosis of pneumonia as an alternative to chest radiography $(21,22)$. In the author's institution, daily lung ultrasound assessment of all PARDS patients allows early detection of new consolidation, improvement in lung recruitment and extravascular lung water assessment without the need for daily chest radiographs; this has both safety and financial benefits.

Finally, ventilator-induced lung injury must be considered. Lung POCUS has been shown to provide accurate assessment of lung compliance and aeration following lung recruitment manoeuvres in both adult and pediatric patient populations $(23,24)$. In a recent case report, lung ultrasound was used to assess a 3-month-old infant with severe ARDS before, during, and after recruitment manoeuvres (24). Lung ultrasound was performed beforehand, and 12 lung regions were reviewed and each region classified into 4 categories as per the ultrasound pattern of lung aeration described by Lichtenstein $e t$ al., and later Bouhemad et al. $(6,23)$.

(I) Normal aeration;

(II) Multiple, irregularly spaced B lines (moderate loss of aeration);

(III) Multiple coalescent B lines (severe loss of aeration);

(IV) Consolidation.

The recruitment manoeuvre consisted of transiently increasing the positive end expiratory pressure (PEEP) in steps of 5 to 20,25, 30 and $35 \mathrm{cmH}_{2} \mathrm{O}$ and holding at each level for 2 minutes. Ultrasound was repeated in real-time during each increment in PEEP with priority given to the posterior, basal regions with the worst aeration. PEEP was then gradually reduced whilst monitoring oxygenation and dynamic lung compliance. The ideal PEEP was titrated at $16 \mathrm{cmH}_{2} \mathrm{O}$, which correlated with an increase in dynamic lung compliance from 2.6 to $3.6 \mathrm{~mL} / \mathrm{cmH}_{2} \mathrm{O}$. These values correlated with lung aeration patterns and CT equivalence (24). The infant remained haemodynamically 


\begin{tabular}{|c|c|c|c|c|c|}
\hline \multicolumn{3}{|c|}{ Quantification of reaeration } & \multicolumn{2}{c|}{ Quantification of loss of aeration } \\
\hline 1 point & 3 points & 5 points & 5 points & 3 points & 1 point \\
\hline B1 $\rightarrow \mathrm{N}$ & B2 $\rightarrow \mathrm{N}$ & $\mathrm{C} \rightarrow \mathrm{N}$ & $\mathrm{N} \rightarrow \mathrm{C}$ & $\mathrm{N} \rightarrow \mathrm{B} 2$ & $\mathrm{~N} \rightarrow \mathrm{B} 1$ \\
\hline $\mathrm{B} 2 \rightarrow \mathrm{B} 1$ & $\mathrm{C} \rightarrow \mathrm{B} 1$ & & & $\mathrm{~B} 1 \rightarrow \mathrm{C}$ & $\mathrm{B} 1 \rightarrow \mathrm{B} 2$ \\
\hline $\mathrm{C} \rightarrow \mathrm{B} 2$ & & & & & $\mathrm{~B} 2 \rightarrow \mathrm{C}$ \\
\hline
\end{tabular}

$\mathrm{N}=$ Normal pattern (normal lung aeration)

$\mathrm{B} 1=$ Multiple well-defined $\mathrm{B}$ lines (moderate loss of lung aeration)

B2 $=$ Multiple coalescent B lines (severe loss of lung aeration)

$\mathrm{C}=$ Lung consolidation

Score calculated by classifying lung aeration (N,B or $C$ ) in each of the 12 lung regions before and after the

applications of PEEP. The sum of the scores in each of these areas is the total ultrasound lung reaeration score.

Figure 8 Ultrasound reaeration score (23).

stable throughout, and subsequently improved with reduction in oxygen requirement and mean airway pressure over the next few hours, and was extubated 5 days following the recruitment manoeuvres (24).

Whilst this was an individual case report, it demonstrates that lung POCUS guided recruitment manoeuvres can be used safely, and without exposure to ionizing radiation in PARDS. Furthermore, this application of POCUS could be used to guide ventilation strategies, and respond more promptly to changes in lung compliance in order to optimise PEEP or plateau pressures. However, one drawback of lung POCUS is the difficulty in detecting lung hyperinflation (24). This limitation can be countered by implementing lung protective strategies in accordance to PALICC recommendations.

We believe lung POCUS can be used to diagnose, manage, and arguably limit some of the complications associated with PARDS.

\section{Lung POCUS in the monitoring of ARDS progression/resolution}

Lung POCUS can also be used in monitoring the lungs of a patient with PARDS for either progression or resolution.

In a prospective study of 40 adult patients with acute lung injury (ALI)/ARDS bedside lung ultrasound was compared to the pressure-volume curve method to assess PEEP-induced lung recruitment. Twelve lung regions were examined and 4 lung ultrasound entities defined as described in the previous section.

The 12 lung regions were examined in PEEP $0 \mathrm{cmH}_{2} \mathrm{O}$ and PEEP $15 \mathrm{cmH}_{2} \mathrm{O}$ and based on the changes in pattern of each lung region an ultrasound reaeration score was produced (Figure 8) (23). The findings demonstrated that an ultrasound reaeration score of $\geq+8$ correlated with PEEP-induced lung recruitment greater than $600 \mathrm{~mL}$, compared to a score of $\leq+4$ being associated with lung recruitment between $75-450 \mathrm{~mL}$ (23). The results also produced "a statistically significant correlation between LUS reaeration score and PEEP-induced increase in $\mathrm{PaO}_{2}$ (Rho $=0.63 ; \mathrm{P}<0.05)$." Furthermore, there was a statistically significant correlation between pressure-volume curve and ultrasound reaeration score measurement of PEEP-induced lung recruitment (Rho $=0.88 ; \mathrm{P}<0.0001)$. This study demonstrated how lung ultrasound can be accurately used to assess lung recruitment and monitor treatment (23).

The previously described case report of a 3-month-old infant with ARDS produced similar findings and promoted the use of lung ultrasound guided recruitment as a "low-cost, easy-to-use diagnostic tool for the pediatric intensivist" (24).

However, as previously mentioned, the limitation of ultrasound in this scenario is the inability to detect hyperinflation. Despite this, the ease of use and ability to perform serial, quick assessments to review and guide bedside management in a pediatric patient with ARDS is promising and warrants larger, randomised-controlled trials.

\section{Cardiac POCUS in ARDS}

It is important to rule out structural heart disease as a possible cause of the respiratory distress. For example, obstructed total anomalous pulmonary venous drainage needs to be considered when dealing with a neonate with suspected ARDS or a large left to right shunt in an infant. However, this would require a structural heart disease echocardiography.

Cardiac POCUS is still helpful to the pediatric intensivist to:

* Assess myocardial function and rule out ventricular 
failure;

* Assess volume status (often to prevent iatrogenic volume overload in the authors experience and assist with diuresis);

* Assess the pulmonary arterial pressures;

* Monitor response to inhaled nitric oxide, if used.

\section{Research needed in POCUS and ARDS}

The evidence that exists for the use of POCUS in ARDS is promising, however there is a paucity of pediatric specific evidence. We need international collaboration to help formalise the diagnostic lung POCUS criteria for PARDS, and how its role can help in the management. Many exciting questions still exist that should be answered: PARDS and alveolar fluid volume status is an area we are currently working on. The addition of cardiac POCUS findings in PARDS would be interesting.

\section{Conclusions}

The adult literature clearly demonstrates that lung POCUS is a safe and validated tool, which is highly sensitive and specific when compared to chest radiography for differentiating the causes of respiratory failure $(4,6,8,10,19)$. Whilst pediatric specific data is limited, it has been shown that the signs seen in adults are reproducible in critically ill neonates and children. Furthermore, we have demonstrated that POCUS can be used not only to diagnose pediatric ARDS, but also to assess for complications, monitor progression and thus guide management. It is clear that high quality, pediatric specific data is required into the use of this exciting imaging modality in PARDS. We strongly recommend that practitioners working with critically ill children attend validated courses in POCUS to allow a governance structure to support this incredibly helpful tool (25).

\section{Acknowledgments}

None.

\section{Footnote}

Conflicts of Interest: The authors have no conflicts of interest to declare.

Ethical Statement: The authors are accountable for all aspects of the work in ensuring that questions related to the accuracy or integrity of any part of the work are appropriately investigated and resolved.

\section{References}

1. Marin JR, Abo AM, Arroyo AC, et al. Pediatric emergency medicine point of care ultrasound: Summary of Evidence. Crit Ultrasound J 2016;8:16.

2. Ord HL, Griksaitis MJ. Fifteen-minute consultation: Using point of care ultrasound to assess children with respiratory failure. Arch Dis Child Educ Pract Ed 2019;104:2-10.

3. Howard ZD, Noble VE, Marill KA, et al. Bedside ultrasound maximises patient satisfaction. J Emerg Med 2014;46:46-53.

4. Saigal S, Joshi R, Sharma JP. Lung ultrasound and Blood Gas-Based Classification of Critically Ill Patients with Dyspnoea: A Pathophysiological Approach. Indian J Crit Care Med 2018;22:789-96.

5. Xirouchaki N, Kondili E, Prinianakis G. Impact of lung ultrasound on clinical decision making in critically ill patients. Intensive Care Med 2014;40:57-65.

6. Lichtenstein D, Goldstein I, Mourgeon E. Comparative Diagnostic Performances of Auscultation, Chest Radiography, and Lung Ultrasonography in Acute Respiratory Distress Syndrome. Anesthesiology 2004;100:9-15.

7. Pereda MA, Chavez MA, Hooper-Miele CC, et al. Lung ultrasound for the diagnosis of pneumonia in children: a meta-analysis. Pediatrics 2015;135:714-22.

8. Lichtenstein DA, Mezière GA. Relevance of lung ultrasound in the diagnosis of acute respiratory failure: the BLUE protocol. Chest 2008;134:117-25.

9. Pediatric Acute Respiratory Distress Syndrome: Consensus Recommendations From the Pediatric Acute Lung Injury Consensus Conference. PALICC, Pediatr Crit Care Med 2015;16:428-39.

10. Baston C, Eoin West T. Lung Ultrasound in Acute Respiratory Distress and Beyond. 2016. J Thorac Dis 2016;8:E1763-6.

11. Lichtenstein DA. Lung ultrasound in the critically ill. Ann Intensive Care 2014;4:1.

12. Mayo P. Bedside pleural ultrasonography: Equipment, technique, and the identification of pleural effusion and pneumothorax. Up-to-date 2018.

13. Copetti R, Soldati G, Copetti P. Chest sonography: a useful tool to differentiate acute cardiogenic pulmonary 
edema from acute respiratory distress syndrome. Cardiovasc Ultrasound 2008;6:16.

14. Wang J, Loh SW, Lee JH. Paediatric acute respiratory distress syndrome: progress over the past decade. J Emerg Crit Care Med 2018;2:24.

15. Huang D, Ma H, Xiao Z, et al. Diagnostic value of cardiopulmonary ultrasound in elderly patients with acute respiratory distress syndrome. BMC Pulm Med 2018;18:136.

16. Wong JJ, Jit M, Sultana R, et al. Mortality in Pediatric Acute Respiratory Distress Syndrome: A Systematic Review and Meta-Analysis. J Intensive Care Med 2019;34:563-71.

17. Khemani RG, Smith LS, Zimmerman JJ, et al. Pediatric Acute Lung Injury Consensus Conference Group. Pediatric acute respiratory distress syndrome: definition, incidence, and epidemiology: proceedings from the Pediatric Acute Lung Injury Consensus Conference. Pediatr Crit Care Med 2015;16:S23-40.

18. Cheifetz IM. Pediatric ARDS. Respir Care 2017;62:718-31.

19. Ebrahimi A, Yousefifard M, Mohammad Kazemi H, et al. Diagnostic accuracy of chest ultrasonography versus chest radiography for identification of pneumothorax: a systematic review and meta-analysis. Tanaffos 2014;13:29-40.

20. Iorio G, Capasso M, De Luca G, et al. Lung ultrasound in the diagnosis of pneumonia in children: proposal for a new diagnostic algorithm. PeerJ 2015;3:e1374.

21. Copetti R, Cattarossi L. Ultrasound diagnosis of pneumonia in children. Radiol Med (Torino) 2008;113:190-8.

22. Jones BP, Tay ET, Eliskashvili E, et al. Feasibility and safety of substituting lung ultrasonography for chest radiography when diagnosing pneumonia in children: a randomized controlled trial. Chest 2016;150:131-8.

23. Bouhemad B, Brisson H, Le-Guen M, et al. Bedside Ultrasound Assessment of Positive End-Expiratory Pressure Induced Lung Recruitment. Am J Respir Crit Care Med 2011;183:341-7.

24. Sameshima YT, Lourenço de Almeida JF, Silva MM, et al. Ultrasound guided lung recruitment in a 3 month old infant with acute respiratory distress syndrome. Ultrasound Q 2014;30:301-5.

25. Griksaitis MJ, Raffaj D, Stephens J, et al. Children's Acute Ultrasound (CACTUS) training: the development of a point of care ultrasound curriculum for pediatric critical care in the UK. Pediatr Crit Care Med 2018;19:67-8.
Cite this article as: Potter SK, Griksaitis MJ. The role of point-of-care ultrasound in pediatric acute respiratory distress syndrome: emerging evidence for its use. Ann Transl Med 2019;7(19):507. doi: 10.21037/atm.2019.07.76 